\title{
LOS JÓVENES Y LA EDUCACIÓN PARA LA CULTURA DE LA SEGURIDAD VIAL
}

\section{THE YOUTH AND THE EDUCATION FOR ROAD SAFETY CULTURE}

\author{
Pilar Baptista Lucio \\ Jorge Reyes Iturbide
}

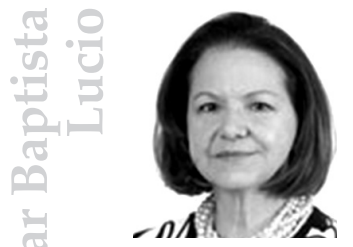

Doctora en Sociología, Universidad Estatal de Michigan. Su investigación se ha centrado en el estudio de los medios y tecnologías de la comunicación, su impacto en las organizaciones y en las personas. Pertenece al Sistema Nacional de Investigadores, nivel I.

Correo electrónico: [pilarbaptista@gmail.com].

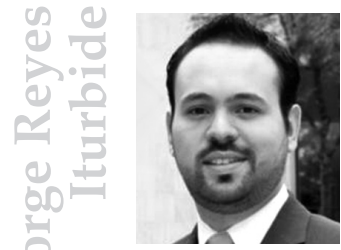

Maestro en Economía y Negocios, y Licenciado en Negocios Internacionales, Universidad Anáhuac. Doctorando en Innovación y Responsabilidad Social, Universidad Anáhuac. Especialidad en Negocios Responsables, Universidad de Nottingham, Inglaterra. Director del Centro IDEARSE para la Responsabilidad y Sustentabilidad de la Empresa, Universidad Anáhuac y co-fundador de la Cátedra Daimler -Anáhuac para la Cultura y Educación Vial, en el marco de la cual se hizo el presente estudio.

Correo electrónico: [jreyes@anahuac.edu.mx].

\section{RESUMEN}

El artículo establece que la cultura vial es un asunto pedagógico, al indagar sobre el tema de la seguridad vial del joven conductor, peatón y pasajero. Con una encuesta a 150 universitarios que actualmente circulan por las calles de varias ciudades mexicanas, se preguntó sobre 
sus patrones de manejo y actitudes hacia normas y medidas de seguridad vial. Se perfila en las respuestas, acatamiento a ciertos reglamentos y señalizaciones, pero también habituales descuidos y conductas inseguras que dan lugar a los incidentes de tránsito y explican la alta ocurrencia de accidentes en nuestro país, un 25 por ciento causados por jóvenes. Se aprecia la necesidad de educar en cultura vial (educación formal), de certificar al conductor rigurosamente (educación no formal) y de implementar campañas mediáticas (educación informal); los mismos sujetos de este estudio señalan que tienen más impacto los mensajes que explicitan las consecuencias de una mala decisión, que aquellos que abordan, desde el humor, el tema de los accidentes viales.

Palabras clave: Jóvenes; educación; cultura vial.

\section{ABSTRACT}

The article argues the pedagogical dimension of Road Safety Education. Exploring a concrete reality, it applied a survey to 150 university students that currently are drivers, passengers or pedestrians in the streets of Mexico. The survey had close -ended questions to elicit information about driving patterns and attitudes towards the road environment and driving, under the premise that attitudes impact our driving. Results show compliance to certain norms and procedures, but also many neglected rules and frequent unsafe behaviors that might lead to road casualties and explain the high incidence of road accidents in our country, where $25 \%$ are caused by young drivers. There is a need to educate and develop a strong road safety culture. The same respondents considered that explicit messages about the consequences of traffic accidents are more effective than those that make jokes about it.

Key words: Youth and education for road safety.

\section{INTRODUCCIÓN}

La cultura vial es un indicador del desarrollo de un país y un tema de educación ciudadana. Atañe a la pedagogía - la escolarizada y la informal- y es además un tema pendiente. México es considerado 
como uno de los países más atrasados en cultura, educación y seguridad vial, según cifras de la Organización Mundial de la Salud ${ }^{1}$. Otros países imparten materias obligatorias sobre seguridad vial; en el nuestro, se ignora el problema en la escuela y en los contenidos mediáticos que pueden considerarse como la otra educación. Las escasas campañas televisivas, contrastan desproporcionadamente con el número de percances calculados en 400000 accidentes viales al año. Para el propósito de este estudio preocupa sobre todo, el 25 por ciento de los conductores implicados en dichos percances y quienes transitan por una etapa de desarrollo que, como señalan Fernández, Callejo, Ibáñez y Vidal²,

[...] se caracteriza por una tendencia a trasgredir las reglas, a tener poca conciencia ciudadana y una alarmante falta de respeto a las normas de tráfico y medidas de seguridad vial, poco respeto por la vida propia y la de los demás.

A los accidentes se les suele clasificar como ocurridos por "condición insegura» (señalización inexistente, pavimento resbaloso, el automóvil tiene defecto de fábrica) o como «acto inseguro», implicando que una conducta individual como manejar bajo el influjo del alcohol, el pasarse los altos e ir a gran velocidad, el no utilizar el cinturón de seguridad, contestar llamadas del teléfono móvil y hasta «textear», son las causas del accidente.

En México, el 90 por ciento de los accidentes de tránsito son resultado de «actos inseguros», es decir, suceden por la imprudencia de alguien; luego, podrían haber sido evitados. Cabe preguntarnos en este espacio, ¿no debe ser éste un tema primordial en la educación? La temática puede incluso insertarse dentro de la Reforma Educativa 20083, en donde en los campos formativos se plasma la aspiración de formar

1 DOMÍNGUEZ, J.C. La inseguridad vial en México, p. 7 quien reporta que, en términos de cultura vial, comparando la OMS a 178 países, México obtuvo calificaciones que dejaban mucho qué desear. En rango del 0 al 10, obtuvo 3 en respeto a límites de velocidad, 3 en ingestión del alcohol, y 5 en cumplimiento de los reglamentos de tránsito.

2 FERNÁNDEZ, M. R., et.al., Análisis de la sensibilización de los jóvenes, p. 307.

3 N.A. Para dichos espacios curriculares diseñamos unas Secuencias didácticas para el niño peatón y el niño pasajero, que buscan incidir en el futuro conductor. 
ciudadanos responsables y con diversas competencias. Específicamente desde preescolar se hace énfasis en «el desarrollo físico y salud», y se describe así la competencia esperada: Practica medidas básicas preventivas y de seguridad para preservar su salud, así como para evitar accidentes y riesgos en la escuela y fuera de ella. Y en la primaria, dentro del campo formativo «Desarrollo personal y para la convivencia», está adscrita la materia «Formación Cívica y Ética» que, entre sus propósitos, busca que los niños comprendan que hay criterios reglas y convenciones externas que regulan su conducta ${ }^{4}$.

Es éste un tema pertinente para la educación y relevante para la sociedad, cuando investigadores de la Cátedra Daimler-Anáhuac ${ }^{5}$ han estimado que

[...] hacia 2015 habrá 6.6 millones de nuevos conductores potenciales y hacia 2020 habrá otros 4.5 millones más. Ambos grupos representarán el 15 por ciento del total de los conductores potenciales en el año 2020, pero son los que podrían tener mayor impacto sobre el número de accidentes y mayor efecto multiplicador desde el punto de vista intergeneracional.

Un primer paso hacia la educación vial, fue explorar las actitudes de jóvenes que hoy en día están conduciendo. Las actitudes se definen -desde el trabajo pionero de Gordon Allport ${ }^{6}$-, como las disposiciones psicológicas que indican la tendencia conductual del sujeto, en este caso hacia un conjunto de situaciones. En el presente estudio, son actitudes hacia el manejo, el evitar accidentes y el respeto a las señales de tránsito, como peatones y pasajeros. Las actitudes - expresadas como opiniones, como es el caso de cualquier investigación por encuestaposeen componentes cognitivos («sé que el cinturón de seguridad impide que me estrelle en el parabrisas»); componentes afectivos («odio utilizar el cinturón en la parte de atrás, me siento atrapado») y componentes activos («si voy a dos cuadras de mi casa, no uso el cinturón»). Al estudiarlas, sabemos que no reportamos la conducta en sí, pero sí

4 SEP, Acciones para la articulación curricular, p. 10 y SEP, Formación Cívica y Ética. 2008, p 67.

5 DOMÍNGUEZ, J.C. Inseguridad vial en México, p. 12.

6 Fue G.W. Allport, profesor norteamericano en Harvard, quien primero conceptualizó y formuló una teoría sobre las actitudes, publicada originalmente en 1935 en el Handbook of Social Psychology, Clark University Press, Worcester, Mass. 
las predisposiciones del sujeto a reaccionar de determinada manera. Bajo las consideraciones anteriores, la pregunta central de esta investigación descriptiva y exploratoria es, ¿cuáles son las actitudes de los jóvenes universitarios ante la seguridad vial?

\section{MARCO CONTEXTUAL : UN PANORAMA DRAMÁTICO}

Antes de responder a dicha pregunta, situémosla en el contexto mexicano, reportando que desde el punto de vista económico, Carlos Domínguez Virgen estima 400 mil accidentes anuales, estimando un costo equivalente a 1.43 por ciento del Producto Interno Bruto (PIB) ${ }^{7}$, cifra que calcula el costo de los daños materiales, el costo de la atención médica y la pérdida de años productivos debido a lesiones y fatalidades por parte de conductores, peatones y otros afectados directamente por los accidentes viales. Lo más preocupante de los accidentes no radica solamente en las pérdidas económicas, sino en las pérdidas humanas. Por ejemplo, el autor establece que es la segunda causa de orfandad en los niños y la segunda causa de muerte hasta los 14 años. En publicaciones posteriores, Carlos Domínguez ${ }^{8}$ indica que en nuestro país, los accidentes ocurren principalmente en áreas urbanas y suburbanas; el 88 por ciento son causados por conductores; sólo 2.1 por ciento es causado por peatones y en un 25 por ciento involucran a conductores adolescentes.

Se reflexiona sobre el carácter multifactorial del problema: falta de reglamentación, se podría empezar un cambio, modificando la edad y los requisitos para la obtención de licencias. En torno a la ingestión del alcohol, sustancia implicada en el 60 por ciento de los accidentes fatales,

[...] las regulaciones existentes son cercanas a los estándares internacionales, aunque muestran algunas fallas, por ejemplo, que el límite máximo en la sangre no es de $0.05 \mathrm{~g} / \mathrm{dl}$ sino de $0.08 \mathrm{~g} / \mathrm{dl}^{9}$.

7 DOMÍNGUEZ, C. La inseguridad vial en México, p. 1. Estima 1.43 por ciento, equivalente a más de 15 mil millones de dólares. Este cálculo se basa en el método de producción perdida.

8 DOMÍNGUEZ, C. Accidentes viales, cultura vial y algunos patrones regionales en el caso de México. p. 3.

9 DOMÍNGUEZ, C. La inseguridad vial en México, p. 60. 
Otro factor importante es el incumplimiento de normas y reglamentos, y en esta incapacidad de vigilancia y aplicación de las sanciones correspondientes, reside nuestra gran debilidad, ya sea con el alcoholímetro o el uso del casco para motociclistas, o con los cinturones de seguridad y asientos especiales para bebés y niños. Pero es en el factor educativo, que promueve el auto-cumplimiento de normas y conductas seguras, donde se inserta la cultura vial construida en la convicción de que cada uno es responsable por su vida y la de los demás. La cultura vial requiere del esfuerzo de todos: una educación desde la escuela - para ser mejor conductor, pasajero y peatón-y desde el hogar, en donde las conductas de los padres (correctas e incorrectas) se tornan en modelos a imitar para los hijos.

Es éste un tema donde se entretejen los términos diferenciados del campo pedagógico: el de la educación formal en la escuela y el de la educación no formal, que estaría situada en el estudio, los exámenes y la certificación de la licencia para conducir que, en nuestro país, es un proceso poco riguroso, tratándose de un tema tan serio: el conductor debe conocer los reglamentos, las señalizaciones y otros temas relacionados con su propia seguridad, la de otros pasajeros y la de los peatones.

Y finalmente, es un tema de cultura, la que se adquiere con la educación informal, con la experiencia cotidiana, con los mensajes de los medios y la influencia paternal. Con el objeto de obtener la «materia prima» para elaborar mensajes didácticos y publicitarios que incidan en la difusión de una cultura vial, se llevaron a cabo las estrategias metodológicas descritas a continuación.

\section{MÉTODO}

Se hizo una investigación por encuestas, exploratoria y descriptiva. Se trata de un estudio preliminar que nos proporciona información sobre aspectos de la cultura vial del universitario del área metropolitana, al indagar sobre sus actitudes hacia el tema. La encuesta fue auto administrada vía Internet, y se contestó durante el mes de octubre de 2012. El instrumento se elaboró con la herramienta para formularios de Google Docs. Una primera versión se piloteó con 25 alumnos. La versión final ya corregida, se subió a Internet y el enlace se envió a profesores de 
seis instituciones de educación superior a quienes se les escribió explicando el motivo e importancia del estudio, pidiéndoles instaran a sus alumnos a contestar de forma anónima (posteriormente a los docentes se les enviaron los resultados para discutirlos en clase).

Esta muestra de conveniencia y de respuesta voluntaria a la encuesta, estuvo compuesta por 150 jóvenes universitarios, matriculados en distintas carreras de universidades privadas (70 por ciento) y públicas (30 por ciento) del área metropolitana de la Ciudad de México. Contestaron a la encuesta 40 por ciento hombres y 60 por ciento mujeres, inscritos en pregrado ( 88 por ciento) y grado (12 por ciento), con un rango de edad entre los 17 ( 2 por ciento) y 26 años (14 por ciento), situándose el 84 por ciento de los sujetos entre los 18 y 25 años cumplidos. El instrumento de 50 ítems puede dividirse en 4 áreas de indagación en torno a la cultura y seguridad vial.

En primer lugar, se preguntó sobre actitudes (tendencias conductuales) de los sujetos como conductores, peatones y pasajeros. Se preguntó además por su respeto a las señalizaciones de tránsito y si atienden otras cosas mientras manejan (uso de celular, «textear», arreglarse, comer, etcétera).

El segundo tipo de preguntas, se dirigió a las opiniones sobre el porqué ocurren tantos accidentes de tránsito. Los sujetos se pronunciaban en qué tan acuerdo estaban con frases que atribuían las causas de los accidentes a situaciones externas (ajenas a ellos) o internas (culpa propia).

En el tercer bloque, se preguntó sobre el tipo de mensaje más efectivo para la prevención de accidentes. Finalmente, se pidieron datos personales, a qué edad obtuvieron su permiso o licencia de conducir, y si ellos o sus familiares habían vivido un accidente vial ${ }^{10}$.

10 Interesante pregunta en el estudio de FERNÁNDEZ, M.R., et. al., Análisis de la sensibilización de los jóvenes, p. 321. 


\section{RESULTADOS}

Tabla 1. ¿Qué tipo de conductor te consideras?

$n=150$

\begin{tabular}{|l|c|c|}
\hline Muy experimentado, atrevido y veloz. & 10 & $7 \%$ \\
\hline Experimentado y confiable, con precaución. & 71 & $47 \%$ \\
\hline Muy precavido con un poco de susto y aprensión. & 33 & $22 \%$ \\
\hline $\begin{array}{l}\text { Tranquilo, calmado, con la filosofía de } \\
\text { «más vale llegar tarde que nunca». }\end{array}$ & 35 & $23 \%$ \\
\hline
\end{tabular}

Para esta pregunta, los sujetos eligieron la descripción que más se ajustara a su percepción como conductor. No es sorprendente que se sitúe más de la mitad como «conductor experimentado» ya que el 61 por ciento obtuvo permiso de conducir antes de los 18 años, edad reglamentaria para obtener una licencia; incluso el 17 por ciento reporta haber obtenido un permiso previo a la licencia a los 15 años o menos.

Tabla 2. Cuando me subo a un automóvil me pongo el cinturón de seguridad $(n=150)$

\begin{tabular}{|l|c|c|c|}
\hline & Siempre & A veces & Nunca \\
\hline Si soy el conductor. & $93 \%$ & $3 \%$ & $2 \%$ \\
\hline Si soy el copiloto. & $85 \%$ & $12 \%$ & $1 \%$ \\
\hline Si voy en los asientos de atrás. & $22 \%$ & $47 \%$ & $29 \%$ \\
\hline Cuando hago un trayecto largo. & $80 \%$ & $15 \%$ & $3 \%$ \\
\hline Uso el cinturón aun en un trayecto muy corto. & $63 \%$ & $22 \%$ & $14 \%$ \\
\hline $\begin{array}{l}\text { Si soy el conductor, pido a todos los pasajeros } \\
\text { que se pongan el cinturón de seguridad. }\end{array}$ & $45 \%$ & $34 \%$ & $18 \%$ \\
\hline
\end{tabular}


En general, se perfila en las respuestas de los sujetos el uso extendido del cinturón de seguridad como piloto (93 por ciento) y copiloto (85 por ciento), y se aprecia como algo ya asumido. Sin embargo, las cifras sombreadas denotan áreas que deben reforzarse de manera puntual e insistente, como el tema de usar el cinturón para cualquier trayecto, en todo momento, y en cualquier parte del vehículo donde se vaya sentado.

Asimismo, observamos en la tabla 3, un acatamiento a las señalizaciones de tránsito, en semáforos e intersecciones (90 por ciento); empero, el disminuir la velocidad en semáforo amarillo o respetar el limite de velocidad en ciertas zonas, solamente 4 de cada 10 jóvenes que contestaron el cuestionario, lo hace habitualmente. También se observa que únicamente 4 de cada 10, cruza las calles por los pasos peatonales. Es justamente en estas categorías de conductas no recurrentes, «a veces» $\mathrm{y}$ «nunca», donde los accidentes suceden. En el estudio preliminar sobre la Inseguridad Vial en México de C. Domínguez (2011), se reportó como causa de accidentes el poco respeto por normas y señalamientos de tránsito. Puede decirse que los jóvenes de esta muestra se han sensibilizado a ciertas disposiciones básicas, pero otras distan de ser acatadas como se esperaría.

Tabla 3. Respeto a las señalizaciones y semáforos ( $n=150)$

\begin{tabular}{|l|c|c|c|}
\hline Respeto las señalizaciones de: & Siempre & A veces & Nunca \\
\hline Alto en semáforo en rojo. & $89 \%$ & $9 \%$ & $0 \%$ \\
\hline Alto en los cruces peatonales. & $84 \%$ & $14 \%$ & $1 \%$ \\
\hline Alto en la intersección de dos calles. & $64 \%$ & $28 \%$ & $6 \%$ \\
\hline Alto en cruces de ferrocarril, tranvías o metrobús. & $82 \%$ & $14 \%$ & $3 \%$ \\
\hline Límite de velocidad, marcado en diferentes zonas. & $38 \%$ & $51 \%$ & $9 \%$ \\
\hline Límite de velocidad por zona escolar. & $62 \%$ & $33 \%$ & $3 \%$ \\
\hline Disminución de velocidad con semáforo en amarillo. & $43 \%$ & $51 \%$ & $4 \%$ \\
\hline $\begin{array}{l}\text { Disminución de velocidad por lluvia y } \\
\text { condiciones difíciles. }\end{array}$ & $75 \%$ & $19 \%$ & $4 \%$ \\
\hline Prohibición de vuelta en ciertas intersecciones. & $73 \%$ & $24 \%$ & $1 \%$ \\
\hline $\begin{array}{l}\text { Cuando soy un peatón y cruzo únicamente por } \\
\text { pasos peatonales y puentes designados. }\end{array}$ & $43 \%$ & $51 \%$ & $4 \%$ \\
\hline $\begin{array}{l}\text { Cuando soy un peatón, me cruzo corriendo } \\
\text { y escojo el camino más corto. }\end{array}$ & $14 \%$ & $57 \%$ & $28 \%$ \\
\hline
\end{tabular}


El descuido cuando se es peatón es más grave, porque indica que la tendencia conductual de más de la mitad de los encuestados, utiliza solamente «a veces» los pasos peatonales y se cruza la calle corriendo, escogiendo el camino más corto. Con referencia a los Gráficos 1 y 2, observamos que utilizar el casco adecuado cuando se anda en bicicleta ha conseguido adeptos, si consideramos que hace diez años poca gente lo hacía. Sin embargo, no sucede así con la motocicleta, donde se requiere inmediata atención para sensibilizar a los jóvenes de las consecuencias de un accidente, cuando no se lleva el casco.

\section{Gráfico 1. Uso del casco en la motocicleta}

Si me subo a una motocicleta, me pongo un casco

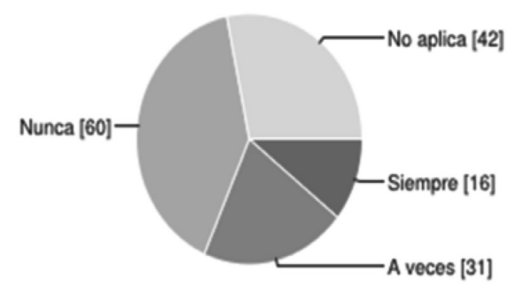

$\begin{array}{lll}\text { Siempre } & 16 & 11 \% \\ \text { A veces } & 31 & 20 \% \\ \text { Nunca } & 60 & 39 \% \\ \text { No aplica } & 42 & 28 \%\end{array}$

Gráfico 2. Uso del casco en la bicicleta

Si ando en bicicleta, utilizo un casco:

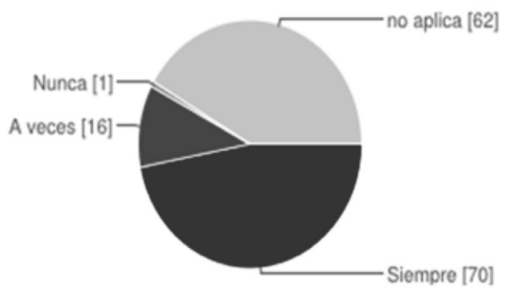

$\begin{array}{lll}\text { Siempre } & 70 & 46 \% \\ \text { A veces } & 16 & 11 \% \\ \text { Nunca } & 1 & 1 \% \\ \text { no aplica } & 62 & 41 \%\end{array}$


En la tabla 4 aparecen marcadas las conductas que declaran los jóvenes hacer mientras manejan y que constituyen un gran peligro para todos. Idealmente, nunca se debería hablar por el celular o «textear», o arreglarse. El manejo requiere total atención, por la velocidad y los factores imprevistos. Sin embargo, el tránsito lento y las conductas multitasking, suponen para muchos «aprovechar el tiempo» en el automóvil, efectuando tareas que suponen un alto riesgo para el conductor y los demás. Hay que agregar que se ha observado que la presencia de la policía de tránsito en las inmediaciones de universidades e institutos, refuerza y concientiza sobre esta falta al reglamento de tránsito.

\section{Tabla 4. Distracciones mientras se conduce $n=150(100 \%)$}

\begin{tabular}{|l|c|c|c|}
\hline & Siempre & A veces & Nunca \\
\hline Escucho las noticias por la radio. & $11 \%$ & $62 \%$ & $25 \%$ \\
\hline Oigo con volumen, mi música favorita. & $72 \%$ & $22 \%$ & $4 \%$ \\
\hline Hago otras cosas, como comer, arreglarme, leer. & $4 \%$ & $45 \%$ & $49 \%$ \\
\hline Hago llamadas de mi teléfono celular. & $9 \%$ & $57 \%$ & $32 \%$ \\
\hline $\begin{array}{l}\text { Utilizo dispositivo «manos libres» } \\
\text { para hacer y recibir llamadas. }\end{array}$ & $29 \%$ & $31 \%$ & $40 \%$ \\
\hline $\begin{array}{l}\text { Manejando, me da tiempo hasta } \\
\text { de mandar mensajes de texto. }\end{array}$ & $3 \%$ & $54 \%$ & $41 \%$ \\
\hline
\end{tabular}

El exceso de velocidad, la invasión de carriles, el no dejar la distancia adecuada con el coche de enfrente, son conductas de riesgo que se potencian con la falta de atención. La concentración debe ser máxima y la música con demasiado volumen, y el uso de audífonos la impiden. ¡Qué decir del uso del celular y de enviar textos! Más del 60 por ciento de los jóvenes de esta muestra declara hacerlo «siempre» o «a veces»; el CESVI reporta, desde su centro de estudios, que en las pruebas realizadas no se observa que se pueda conducir bien y tener una conversación al mismo tiempo ${ }^{11}$. En la tabla 5, se compilaron las frases sobre la explicación que dan otras personas del porqué ocurren tantos accidentes viales y en donde los jóvenes marcaron su acuerdo o desacuerdo a dichas opiniones.

11 Centro de Experimentación y Seguridad Vial México, en: [http:/ / www.cesvimexico.com.mx/]. 


\section{Tabla 5. ¿Por qué ocurren tantos accidentes viales? ( $n=150)$}

\begin{tabular}{|c|c|c|}
\hline \multicolumn{3}{|l|}{$\begin{array}{l}\text { 1. Porque la construcción de vialidades } \\
\text { es pésima y sin lógica: }\end{array}$} \\
\hline En desacuerdo total. & 18 & $12 \%$ \\
\hline Estoy de acuerdo, pues esto es frecuente. & 85 & $56 \%$ \\
\hline Totalmente de acuerdo. & 46 & $30 \%$ \\
\hline \multicolumn{3}{|l|}{$\begin{array}{l}\text { 2. Porque la autoridad se dedica } \\
\text { a extorsionar a los conductores: }\end{array}$} \\
\hline En desacuerdo total. & 27 & $18 \%$ \\
\hline Estoy de acuerdo, pues esto es frecuente. & 63 & $41 \%$ \\
\hline Totalmente de acuerdo. & 59 & $39 \%$ \\
\hline \multicolumn{3}{|c|}{$\begin{array}{l}\text { 3. Porque se dan permisos y licencias de conducir } \\
\text { a quien no sabe manejar: }\end{array}$} \\
\hline En desacuerdo total. & 11 & $7 \%$ \\
\hline Estoy de acuerdo, pues esto es frecuente. & 55 & $36 \%$ \\
\hline Totalmente de acuerdo. & 83 & $55 \%$ \\
\hline \multicolumn{3}{|l|}{$\begin{array}{l}\text { 4. Hay muchos accidentes porque manejamos } \\
\text { a exceso de velocidad: }\end{array}$} \\
\hline En desacuerdo total. & 13 & $9 \%$ \\
\hline Estoy de acuerdo, pues esto es frecuente. & 51 & $34 \%$ \\
\hline Totalmente de acuerdo. & 85 & $56 \%$ \\
\hline \multicolumn{3}{|c|}{$\begin{array}{l}\text { 5. Hay accidentes fatales porque creemos que manejamos } \\
\text { «muy bien», aun tomando unas copas: }\end{array}$} \\
\hline En desacuerdo total. & 12 & $8 \%$ \\
\hline Estoy de acuerdo, pues esto es frecuente. & 30 & $20 \%$ \\
\hline Totalmente de acuerdo. & 107 & $70 \%$ \\
\hline \multicolumn{3}{|l|}{$\begin{array}{l}\text { 6. Hay tantos accidentes porque nos falta } \\
\text { ser ciudadanos con cultura y educación vial: }\end{array}$} \\
\hline En desacuerdo total. & 13 & $9 \%$ \\
\hline Estoy de acuerdo, pues esto es frecuente. & 18 & $12 \%$ \\
\hline Totalmente de acuerdo. & 118 & $78 \%$ \\
\hline
\end{tabular}


Las tres primeras frases se refieren a causas externas al conductor. Las tres últimas a «actos inseguros», es decir a la imprudencia de quien conduce. En personas inmaduras es frecuente encontrar que las causas de cualquier problema se atribuyen a una situación externa a ellos mismos. Aquí, por el contrario, observamos que con mayor incidencia, los jóvenes piensan que los accidentes ocurren por decisiones personales equivocadas. Nótese por ejemplo que el 90 por ciento de ellos está «totalmente de acuerdo» $\mathrm{o}$ «de acuerdo» en que los accidentes ocurren porque tenemos un problema de civilidad, falta cultura y educación vial. De igual manera, una abrumadora mayoría coincide en que las causas de los accidentes tienen origen en el exceso de velocidad; en la Tabla 3, el 60 por ciento admite «no siempre» o «nunca» respetar los límites de velocidad marcados en las señalizaciones.

Grandes paradojas emergen, sin embargo, en el estudio de actitudes, donde cognitivamente se puede estar cierto sobre algo y a la hora de actuarlo no hay coherencia entre lo que sabemos y lo que hacemos. Existe también gran acuerdo (90 por ciento) en que una de las causas de los accidentes de tránsito, es porque creemos que manejamos muy bien aun tomando unas copas, aunque en otra pregunta, solamente el 4 por ciento declaró haber reprobado el alcoholímetro.

De la misma manera, en esta misma muestra de jóvenes que se perciben a sí mismos como conductores experimentados, precavidos y tranquilos (sólo el 7 por ciento se considera atrevido y veloz), encontramos que tienden a no usar el cinturón en trayectos cortos, ni en la parte de atrás del vehículo; asimismo con frecuencia sobrepasan límites de velocidad y hacen caso omiso de ciertas señalizaciones y peor aún, frecuentemente «textean» y van utilizando el celular. Quizás la explicación es que sobrevaloran sus propias habilidades de manejo, confían en la suerte y actúan con base en una sensación de invulnerabilidad ante el peligro. Pero, ¿y su propia experiencia de accidentabilidad vial?

En esta muestra, hallamos que el 72 por ciento de los jóvenes dice haber estado en un accidente automovilístico leve y el 16 por ciento en alguno cuyo resultado fue la pérdida total del vehículo. El 38 por ciento tiene hermanos o familiares que han estado en un accidente grave y 14 por ciento $(n=21)$ reportaron haber perdido a un familiar en un accidente fatal. Sin embargo, por la incidencia de conductas de 
riesgo descritas en esta sección, tal pareciera «que para la sensibilización hacia medidas de seguridad vial, no importa el haber vivido o no un accidente de tráfico» ${ }^{12}$.

En los resultados se van perfilando las líneas en las que se debe trabajar para la educación y la cultura vial (el carácter protocolario -es decir, siempre seguir las reglas - de conducir un vehículo): la reflexión sobre las consecuencias de saltarse dichas reglas, la responsabilidad sobre nuestra seguridad y la de los demás. Ahora bien, ya sea en unos cursos formales o mediáticamente, ¿cómo deben abordarse estos temas? ¿Con detalle científico? ¿Cómo pérdida real de la vida y la salud? ¿Apelando a la responsabilidad? ¿Con sentido del humor? Al describir en el cuestionario seis tipos de mensajes diferentes, esta muestra de jóvenes aconseja cuál es el tono del mensaje que considera de más impacto.

Ordenados para este reporte en la siguiente serie de gráficos, se dispusieron - con base en el mayor número de respuestas agrupadas en mayor impacto - diferentes anuncios televisivos que promueven la cultura vial. Estos mensajes tipo quedan ilustrados para el lector en las descripciones y en los enlaces que hemos puesto al pie de página ${ }^{13}$. Bajo las consideraciones anteriores, tenemos en primer lugar que para el 67 por ciento de la muestra, los mensajes de mayor impacto en su conducta, son aquellas situaciones vívidamente dramatizadas, que muestran las consecuencias reales (niños y adultos muertos, heridas y sangre, desesperación de familiares $)^{14}$. Para el 55 por ciento, el mostrar de manera científica las consecuencias de la combinación de no usar cinturón y velocidad. También con el 55 por ciento, pero con menos frecuencia en la categoría 7, el recordatorio de que se es responsable por la vida de otros, tiene cierto impacto. Las sugerencias y metáforas, y el humor, son considerados los menos efectivos en cuanto a su impacto para educar, recordar o seguir procedimientos de seguridad vial.

12 Hallazgo que también encuentran en España. Cfr. FERNÁNDEZ, M.R., et. al., Análisis de la sensibilización de los jóvenes, p. 319.

13 Se trata de mensajes que también utilizamos para la muestra piloto del estudio, pidiéndoles sus reacciones en un grupo de foco.

14 Ver anuncio de Inglaterra sobre las consecuencias de textear y manejar, en: [http:/ / www. youtube.com/watch?v=R0LCmStIw9E]. 


\section{SERIE DE TEXTOS Y GRÁFICOS. IMPACTO DE MENSAJES SOBRE SEGURIDAD VIAL $\mathbf{N}=150$}

Mensajes que muestran vívida y dramáticamente las consecuencias de los accidentes automovilísticos. Se visualiza una distracción que causa que choquen cuatro vehículos. Se muestran heridos, muertos, niños gritando ("iqué les pasa a sus papás que no responden!") ${ }^{15}$.

\section{Gráfico 3.1. Mensajes vívidos y dramáticos:}

$\begin{array}{lrl}1 \text { - Poco impacto } 1 & 1 \% \\ 2 & 1 & 1 \% \\ 3 & 9 & 6 \% \\ 4 & 10 & 7 \% \\ 5 & 27 & 18 \% \\ 6 & 33 & 22 \% \\ 7 \text { - Gran impacto } & 68 & 45 \%\end{array}$

Mensajes que muestran de una manera científica y con un experimento filmado con dumios o maniquíes, las consecuencias de chocar a $30 \mathrm{~km} /$ hora y no traer el cinturón de seguridad abrochado ${ }^{16}$.

\section{Gráfico 3.2. Mensajes que muestran experimento:}

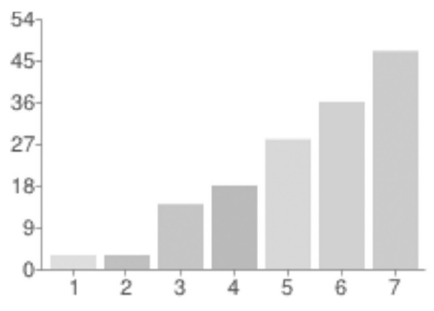

1 - Poco impacto $32 \%$

$2 \quad 32 \%$

$3149 \%$

$4 \quad 1812 \%$

$5 \quad 2818 \%$

$6 \quad 3624 \%$

7 - Gran imbacto $4731 \%$

15 Ver anuncio realizado en Inglaterra sobre las consecuencias de textear y manejar, en: [http:/ / www.youtube.com/watch?v=R0LCmStIw9E].

16 Velocidad y prueba con dummies, en: [http:/ / www.youtube.com/watch?v=AEkV70P15c8]. 
Mensajes que sugieren tu responsabilidad sobre la vida de otros cuando eres conductor. Sin mostrar niños accidentados, ilustran la diferencia de pedir a los pasajeros que se pongan el cinturón en la parte de atrás ${ }^{17}$.

\section{Gráfico 3.3. Mensajes que apelan a la responsabilidad:}

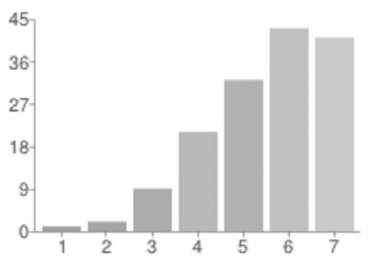

Poco impacto
Gran impacto
1 - Poco impacto 1 1\%

2

$21 \%$

$96 \%$

$2114 \%$

$3221 \%$

$4328 \%$

6

7 - Gran impacto $4127 \%$

Mensajes o consejos sobre seguridad vial que mencionan y muestran a seres queridos. Se enfatiza que la persona sea precavida, porque su familia le espera ${ }^{18}$.

\section{Gráfico 3.4. Mensajes que te recuerdan a los seres queridos:}

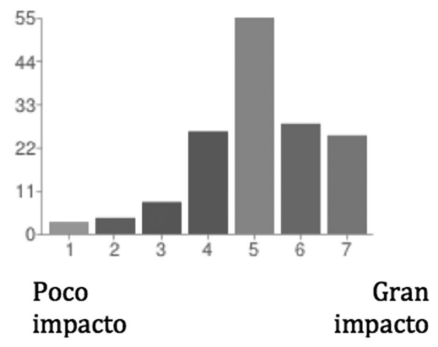

$\begin{array}{lrl}1 \text { - Poco impacto } 3 & 2 \% \\ 2 & 4 & 3 \% \\ 3 & 8 & 5 \% \\ 4 & 26 & 17 \% \\ 5 & 55 & 36 \% \\ 6 & 28 & 18 \% \\ 7 \text { - Gran impacto } 25 & 16 \%\end{array}$

17 Niños con y sin cinturón, en: [http:/ / www.youtube.com/watch?v=KvxGXSGuHbA].

18 Anuncio Embrace Life, en: [http:/ / www.youtube.com/watch?v=VWhiwDh3wbo]. 
Mensajes irónicos, actuados de manera graciosa, donde la policía detiene a un conductor, se baja del auto, da tumbos, arrastra la lengua y dice que «está en perfecto estado» ${ }^{19}$.

\section{Gráfico 3.5. Mensajes irónicos y con humor}

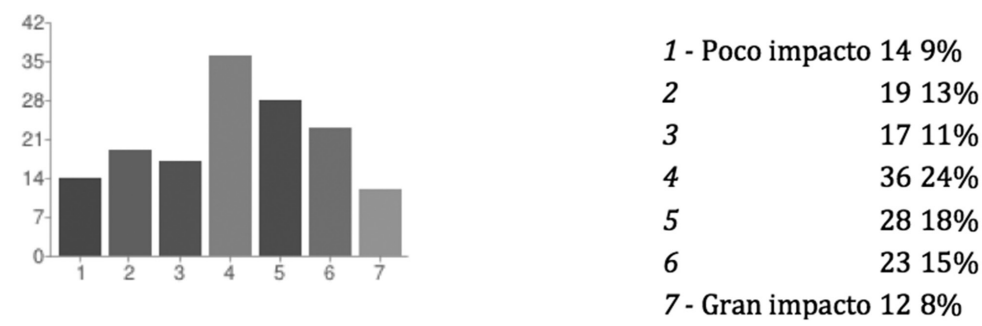

\section{CONCLUSIÓN}

Los resultados presentados denotan una falta de cultura vial que afecta la vida cotidiana de todos. La incidencia de accidentes de tránsito en nuestro país, se explica por la tendencia a conductas inseguras del joven conductor, pasajero o peatón. Aunque con una muestra limitada en número y geografía - y sin descartar en las respuestas a un cuestionario de este tipo, la disposición de los sujetos a contestar lo socialmente deseable-, el estudio ilustra y apunta claramente a aspectos en los que hay que trabajar. Se perfila la necesidad de socialización hacia una cultura vial sólida en donde ser requiere internalizar el carácter del accidente, el que ocurre cuando «a veces» no se acata la regla, Por ejemplo, un doctor no puede operar con guantes esterilizados «a veces» $\mathrm{o}$ «casi siempre», si no sigue siempre un protocolo de asepsia e higiene en el 100 por ciento de las cirugías. En un avión no se puede exigir «frecuentemente» el uso de cinturones de seguridad en el despegue y contemporizar con un porcentaje de pasajeros que «no lo abrocha porque es un trayecto corto».

19 Un ejemplo de este tipo de comercial es el de «Paco Malacopa» en México, en: [http:/ / www.youtube.com/watch?v=nLYT4U6lq4U]. 
Los resultados describen a una muestra de jóvenes que distingue entre lo permitido y lo indebido, que conoce los reglamentos y señalizaciones, y que los acata con cierta frecuencia, pero "no siempre», incurriendo así en conductas de riesgo. ¿Qué factores psicosociales pueden estar interviniendo? ¿Por qué los jóvenes se alejan de un procedimiento y prefieren actuar de manera temeraria e irresponsable? Futuros estudios deben considerar causas subyacentes.

Todos necesitamos respetar los reglamentos de tránsito; las autoridades revisar sus criterios para otorgar permisos y licencias de manejo, además de aumentar la vigilancia para el acatamiento de las normas. Pero, más allá de la prueba del alcoholímetro, o de multas por infracciones, se requiere desde el ámbito educativo - formal, no formal e informal- promover la formación de una cultura vial para toda la vida. Herramientas pedagógicas desde la escuela, la familia, la certificación del conductor y los medios de comunicación deben aportar coincidiendo en la solución del problema. Campañas que concienticen y que expliciten claramente las terribles consecuencias de los errores de quien conduce irresponsablemente y la educación formal que forme en competencias para una conducta insoslayable y con gran repercusión en los otros: la del niño pasajero, el peatón y la del futuro conductor,

Se sugiere también con base en los hallazgos, que para los jóvenes que hoy están manejando, se ofrezcan cursos en la universidad sobre los aspectos señalados, que incluso los prepararán para ser —como padre o madre de familia - un conductor responsable.

\section{REFERENCIAS}

BAPTISTA, P. y REYES, A.L., Secuencias didácticas para la educación del niño peatón y el niño pasajero, Cátedra Daimler Anáhuac. Centro IDEArse para la Responsabilidad y Sustentabilidad de la Empresa, Universidad Anáhuac México Norte, 2011.

CENTRO DE EXPERIMENTACIÓN Y SEGURIDAD VIAL MÉXICO. Estudios, campañas y documentos, en: [http:/ / www.cesvimexico. com.mx/]. 
DOMÍNGUEZ, J.C., Estudio de la inseguridad vial en México: consecuencias económicas y sociales, Informe Final de estudio para la Cátedra Daimler Anáhuac, Centro IDEArse para la Responsabilidad y Sustentabilidad de la Empresa, Universidad Anáhuac México Norte, 2011, 95 páginas.

DOMÍNGUEZ, C., Accidentes viales, cultura vial y algunos patrones regionales en el caso de México, Cátedra Daimler Anáhuac. Centro IDEArse para la Responsabilidad y Sustentabilidad de la Empresa, Universidad Anáhuac México Norte, 2012.

DOMÍNGUEZ, J.C., «Accidentes viales, la nueva epidemia», RSE Ideas, febrero-marzo, Grupo Mundo Ejecutivo, 2013, pp. 8-12.

FERNÁNDEZ, M.R.; CALLEJO, S.; IBÁÑEZ, S. y VIDAL, J.V., «Análisis de la sensibilización de los jóvenes ante la seguridad vial. Bases para la elaboración de un programa», Relieve, Revista electrónica de investigación y evaluación educativa, vol. 12, n. 2, 2006, p. 307324, en: [www.uv.es / RELIEVE/v12n2 / RELIEVEv12n2_7.htm].

KARAISL, M. y DOMÍNGUEZ, J.C., «El problema de los accidentes viales», Bicentenario: el ayer y hoy de México, vol. 5, n. 17, Instituto José María Luis Mora, 2012, pp.

ORGANIZACIÓN PANAMERICANA DE SALUD. Informe sobre el estado de la seguridad vial en la región de las Américas, 2009.

SECRETARÍA DE EDUCACIÓN PÚBLICA, Acciones para la articulación curricular 2006-2012, Subsecretaría de Educación Básica y Normal, SEP, marzo, México, 2008.

SECRETARÍA DE EDUCACIÓN PÚBLICA, Formación Cívica y Ética, Dirección General de Materiales Educativos de la Subsecretaría de Educación Básica, Secretaría de Educación Pública, México 2008.

SECRETARÍA DE EDUCACIÓN PÚBLICA, Guía para la educadora, Educación Preescolar. Dirección General de Materiales Educativos de la Subsecretaría de Educación Básica, Secretaría de Educación Pública, Secretaría de Educación Pública, México, 2009. 